\title{
Numerical Simulation of Turbulent flow in a Channel Containing a
} Small Slot

\author{
Jhon Goulart*¹, Jan G. Wissink ${ }^{2}$, Luiz C. Wrobel ${ }^{2}$ \\ ${ }^{1}$ Group of Experimental and Computational Mechanics - University of Brasilia, Gama, \\ 72.405-610, Brazil \\ ${ }^{2}$ College of Engineering, Design and Physical Science - Brunel University London, \\ Uxbridge, UB8 3PH, UK \\ *Corresponding author. +55 6131078209 \\ E-mail address: jvaz@unb.br (J. Goulart)
}

Abstract: A three-dimensional unsteady simulation was carried out to predict the main features of the turbulent flow inside a closed channel connected to a lateral slot. The incompressible turbulent flow was modelled using a hybrid Detached Eddy $\underline{\text { Simulation }}$ (DES), that uses an LES/URANS approach to predict the turbulence. The calculations were performed using ANSYS $^{\circledR}$ CFX. In this work the main channel has a size of 180 $\mathrm{mm} \times 136.20 \mathrm{~mm}$. The small subchannel is characterized by its deepness, $\mathrm{p}=77 \mathrm{~mm}$ and width, $d=10 \mathrm{~mm}$. The Reynolds number, based on the hydraulic-diameter, $\mathrm{D}_{\mathrm{h}}$, the bulk velocity, and the kinematic viscosity, $v$, in the main channel was $\operatorname{Re}=2.25 \times 10^{5}$. Inside the small slot the velocity distribution was found to depart from the law of the wall and the normal Reynolds stresses, $\overline{u^{\prime} u^{\prime}}$ and $\overline{v^{\prime} v^{\prime}}$, were found to dominate the mixing process. Velocity time-traces extracted at locations as far as $y / p=1.125$ inside the gap evidenced the presence of large eddies travelling inside the small channel. It was shown that periodic streamwise boundary conditions can be applied to this problem, and good results were obtained by using a channel length that was approximately twice the wavelength of the experimentally observed coherent structures. The results were found to be in fair agreement with the results presented in Meyer and Rehme (1994), though a certain lack of information on turbulence in single channels connected to a gap still remains.

Keywords: turbulent flow simulation, compound channel, periodic flow path, gap instability, large scale structure

\section{1 - INTRODUCTION}

Compound channels are found in many branches of engineering. These channels are characterized by the presence of a narrow region connected to one or more wider regions. Double-pipe heat exchangers, assembles of rod bundles in nuclear reactors and 
water supply channels with shallow inundated planes are some examples of applications of compound channels in real life. Also, arteries containing catheters or stents might be thought of as compound channels in biological systems.

The structure of turbulent flow in compound channels is significantly affected by the presence of narrow gaps. The gaps are responsible for a change in mass flux distribution inside the channel producing an inflexional mean velocity field which is a source of instability resulting in turbulence production (Meyer and Rehme, 1994, Goulart and Möller, 2007, Goulart et al., 2013, Home and Lightstone, 2014, Home et al., 2009, Merzari et al., 2008). Unusually high turbulence intensities are measured in the vicinity of the gap. This turbulence intensity is much higher than the usual levels found in pipe flow implying an enhanced mixing between channels (Rehme, 1992).

Secondary flows were once thought to be the main responsible for the high mixing rates that were found in the gap region. However, they were found to be too weak, less than $1 \%$ of bulk velocity, to promote such mixing (Meyer, 2010). Hence, the increased mixing rates in the gap must be due to a different mechanism. In fact, one of the most impressive characteristics of flow in compound channels are the gap instabilities and the turbulence they produce, which according to Meyer (2010), is the true reason for increased mixing rates in the gap region.

Flow pulsations (or "gap instabilities" as recently termed by Tavoularis, 2011) in rod bundles were first reported by Rowe (1974). In his experiments a periodic path in the axial velocity was found in a $3 \times 3$ square-array rod bundle for $\mathrm{P} / \mathrm{D}=1.10$ ( $\mathrm{P}$ is the pitch, distance between the centres of adjacent rods and $\mathrm{D}$ the diameter of the rod). The frequency associated with this phenomenon was found to increase when the distance between the rods was reduced, however, for $\mathrm{P} / \mathrm{D}=1.25$ these alternating motions were found to vanish, thus providing some evidence of the dependence of this instability on the geometric parameters. Since then many experimental works were carried out to study and understand the mixing processes in rod bundles, the oscillating flow characteristics, as well as the dependence on the geometry and Reynolds number e.g. Hooper and Rehme (1984), Möller (1991), Rehme (1992), Wu and Trupp (1993), Rehme and Meyer (1998), Guellouz and Tavoularis (2000 a).

Hooper and Rehme (1984) concluded that mass, momentum and energy in the vicinity of the gap are ruled by an energetic and almost periodic turbulent velocity component, and that there is no relation with mean secondary flow velocities generated by Reynolds stress gradients. A similar conclusion was reached by Möller (1991), who 
applied hot wire anemometry to measure the Reynolds stresses and to determine the origin and characteristics of large eddies in a matrix of 4 rod bundles with P/D ratio ranging from 1.007 up to 1.224. Regions with relatively higher axial turbulence intensity were found at about $25^{\circ}$ from the gap between the rods and $35^{\circ}$ from gap between the rods and channel walls. Furthermore, Möller (1991) concluded that the large vortices originated from instantaneous gradients formed on each side of the gap. A number of years later Guellouz and Tavoularis (2000 a), in their experimental work in a channel containing a single rod, showed that large scale structures are arranged in the form of a vortex street on either side of the gap.

A series of single channels containing lateral slots and twin channels connected by a narrow gap were experimentally studied by Meyer and Rehme (1994, 1995). The main dimensionless geometrical parameters were the depth and the width of the slot, $\mathrm{p}$ and $\mathrm{d}$, respectively. The investigations were performed for several p/d-ratio ranging from 1.00 to 8.28. The amount of turbulent kinetic energy and the intensity of Reynolds stresses generated near the gap were higher than anywhere else in the channel. The kinetic energy was found to be three times stronger than in a square channel. The normal Reynolds stresses were found to be highly anisotropic, implying that URANS models would not be able to accurately describe turbulence in these channels.

The large-scale structures appearing near the gap were also investigated by the authors of both papers. They concluded that large-scale flow oscillation exists for, $\mathrm{p} / \mathrm{d}>2$.

Years later, Goulart and co-workers (Goulart and Möller, 2007 and Goulart et al., 2013) published the outcomes of their experimental research on 10 rectangular closed compound channels showing that the flow inside the channels is prone to the instability due to the inflexional velocity profile. The authors also concluded that the flow is quite similar to a spatial mixing layer formed between the slower fluid (inside the gap) and the faster fluid from main channel. One year later Choueiri and Tavoularis (2014) attributed the "gap vortex street" formation in an eccentric annular channel to the instability of two mixing layers on both sides the gap.

Various numerical codes have been used to investigate the flow in compound channels, mostly in the last decade. Unsteady simulations have been carried out in twin channels by Home et al. (2009), Home and Lightstone (2014) and Derksen (2010), in channels with a single rod by Chang and Tavoularis (2005, 2008, 2012), while Mezari et al. (2008) studied flow in a tight-lattice fuel bundle. All these works aimed to qualify 
and quantify the turbulent flow phenomenology, in some cases by employing various turbulence models.

The thorough work carried out by Chang and Tavoularis (2012) was aimed at understanding the onset of flow gap instabilities in a channel containing a single rod, using the results published in Guellouz and Tavoularis (2000a) as a benchmark. The numerical study was performed using several turbulence models, various inlet conditions (developed and non-developed velocity profiles, as well as transient inflow). Despite the fact that LES produced the most accurate results, URANS also appeared to be suitable to predict turbulent flow characteristics in compound channels. However, the hybrid models (SAS and IDDES) were found to predict an earlier onset of gap instabilities.

Chang and Tavoularis (2012) also identified the presence of a mixing layer in the equidistant plane between the bottom channel and the rod. They concluded that it is acceptable to use URANS to calculate flow in tightly packed rod bundles, and that a uniform inflow velocity is preferable over fully-developed profiles. Also, inlet/outlet boundary conditions should be applied rather than streamwise periodic boundary conditions.

The present work is aimed to investigate numerically the structure of the turbulent flow in a rectangular channel with a lateral slot.

Mean average quantities distributions and the dynamics of the large scale structures, such as the main frequency, Strouhal number, wavelength and the convection velocity were studied in detail. The numerical calculations were carried out using the ANSYS ${ }^{\circledR}$ CFX package using a hybrid (LES/RANS) turbulence modelling approach. The Reynolds number, based on the bulk velocity, $U_{b}$, the hydraulic-diameter, $D_{h}$ and the kinetic viscosity was $\operatorname{Re}=2.25 \times 10^{5}$. To minimise computational costs we decided to apply periodic boundary conditions in the streamwise direction, thereby shortening significantly the domain in comparison with the experimental work performed by Meyer and Rehme (1994). Experimental results of the dynamics of the turbulent flow, which were published in 1994 by Meyer and co-worker, were used for comparison.

\section{2 - COMPUTATIONAL DOMAIN AND MESH}

As illustrated in Fig. 1 (c) the computational domain that was used to carry out the simulation consists of a single main channel with a small subchannel or slot. The main channel is connected to the subchannel by a narrow gap of width $\mathrm{d}=10 \mathrm{~mm}$, whereas 
the depth of the slot is given by $\mathrm{p}=77 \mathrm{~mm}$. The dimensions of the main channel are shown in Fig. 4 (b), where $\mathrm{H}=180 \mathrm{~mm}$ is the height. The cross-section of the channel matches the one studied by Meyer and Rehme (1994). However, the original length of the channel of $7000 \mathrm{~mm}$ was significantly shortened to $730 \mathrm{~mm}$ in this computation. This was made possible by employing periodic boundary conditions in the streamwise direction in combination with a fixed mass flow rate, $\dot{m}=0.5512 \mathrm{~kg} / \mathrm{s}$. The length of the periodic channel was taken to be twice the wavelength provided by Meyer and Rehme in their papers from 1994 and 1995. A no-slip condition was applied at the walls.
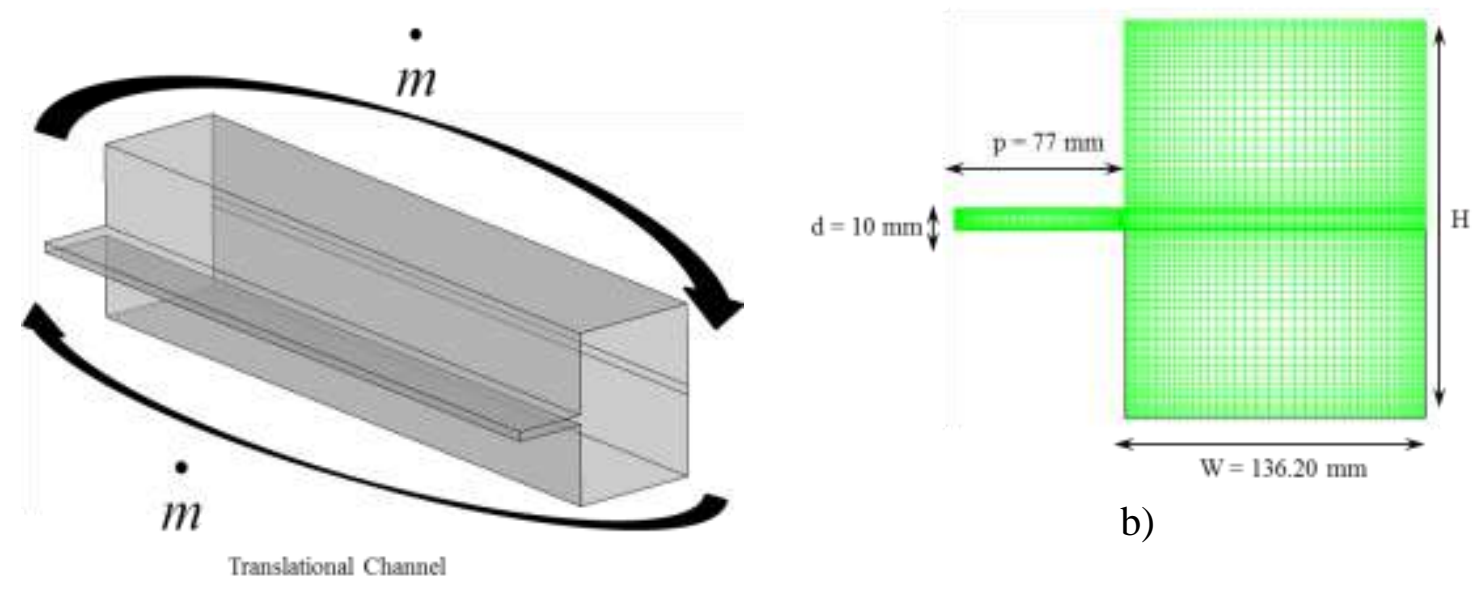

b)

a)

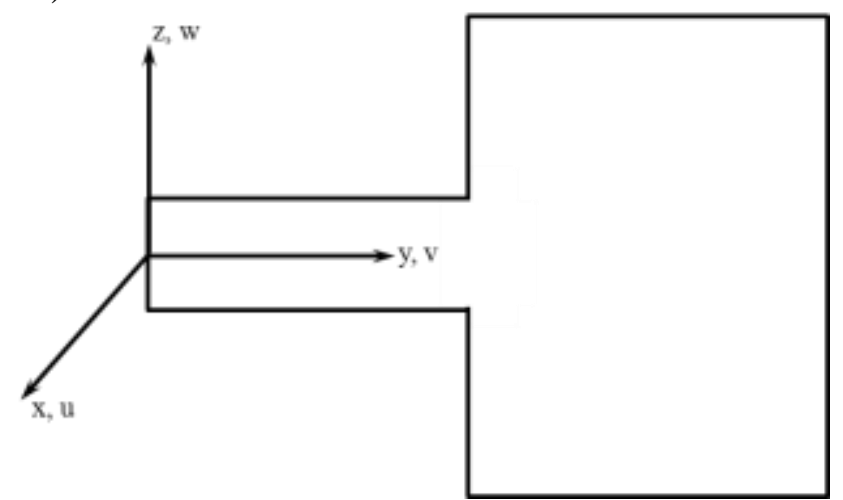

c)

Figure 1 - a) schematic view of compound channel. b) channel's cross-section and its dimensions. c) coordinates and velocity components.

The mesh was built in blocks that allow a discretization of the domain with hexahedral volumes. Such a strategy of building a mesh is appropriate for applying periodic boundary conditions as the inlet/outlet surfaces can be perfectly matched.

Special care was taken with the mesh near the walls inside the small subchannel and at the interface between the main and the small subchannel. Near the subchannel's walls we applied a mesh refinement in order to obtain a $\mathrm{y}^{+}$of about 5 . To capture the gradient 
of the velocity and the strong spanwise velocity at the interface between both channels, the same mesh refinement was applied to the bottom wall of the small channel.

Along the streamwise direction of the channel 100 equidistant grid points were used, yielding a cell size of $\Delta \mathrm{x} / \mathrm{d}=0.73$. A test in which the streamwise cell size was reduced by about $20 \%$ did not lead to any changes in the main or fluctuating fields. At the walls of the main channel, the value of $\mathrm{y}^{+}$ranged between 30 and 70 . During the simulation we worked with three different meshes, which are described in Table 1. Mesh M1 is based on the mesh built by Home and Lightstone (2014) and their study on the suitable mesh and refinements for twin-channels. After analysing the results, mesh M2 was deemed to give sufficiently accurate results and was hence employed for all further simulations. Note that the mesh refinement near the wall mentioned above was only employed in mesh M2 obtaining a y+ of about 5 and an $\mathrm{x}+$ of around 90 at the narrow slot.

Table 1: Meshes used in the present work

\begin{tabular}{ccccccc}
\hline \multicolumn{2}{c}{ Slot $-\mathrm{N} \#$ of divisions } & \multicolumn{3}{c}{$\begin{array}{c}\text { Main Channel }-\mathrm{N} \# \text { of } \\
\text { divisions }\end{array}$} & Total number of nodes \\
\cline { 1 - 4 } Mesh & $\mathrm{p}$ & $\mathrm{d}$ & $\mathrm{H}$ & $\mathrm{W}$ & $\Delta \mathrm{x} / \mathrm{L}$ & \\
name & 63 & 9 & 70 & 55 & 100 & 516000 \\
M1 & 80 & 20 & 70 & 55 & 100 & 599635 \\
M2 & 63 & 9 & 70 & 55 & 120 & 618310 \\
M3 & & & & & & \\
\hline
\end{tabular}

To confirm the quality of the dynamic results we doubled the channel's length (from $730 \mathrm{~mm}$ to $1460 \mathrm{~mm}$ ) using mesh M2. No significant change in the dynamic features of the coherent structures such as frequency, convection velocity and wavelength was found. The main frequency, for instance, only changed marginally from $23.80 \mathrm{~Hz}$ to $23.53 \mathrm{~Hz}$.

During the time-dependent simulation the time step was kept at $\Delta \mathrm{t}=3.35 \times 10^{-5} \mathrm{~s}$, which was small enough to ensure a maximum Courant number lower than one. The total simulation time was about 37 flow-through times. The statistical quantities were averaged over the last 0.85 seconds, corresponding to 25.40 flow-through times, after making sure that the turbulence was stationary. To obtain proper initial conditions, the results of a simulation using inlet-outlet boundaries combined with the k- $\omega$ SST model were used. The inlet condition was prescribed using the data provided by Meyer and Rehme (1994). The turbulent intensity at the inlet (not provided by Meyer and Rehme) was set to $5 \%$. 
The simulation was carried out using the backward Euler scheme for time discretization. A Bounded Central Difference Scheme was employed to solve the advective terms in all equations. A second-order central difference was applied in the LES region (away from the walls), whereas in the URANS region (at the walls) we applied a second-order upwind.

At the walls, the automatic wall treatment feature of CFX was used (Menter et al. 2003). Near the walls this feature automatically shifts from the standard low-Re formulation to wall functions, based on the grid spacing of the near-wall cells.

\section{3- GOVERNING EQUATIONS}

\section{1 - Mass and Momentum Conservation}

In this paper we are modelling turbulence by using the DES-SST hybrid model. This

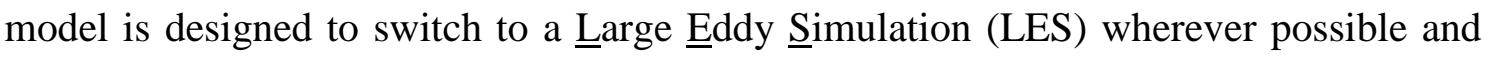
otherwise use the k- $\omega$ SST - URANS model. In the present simulation LES is likely to be employed in the free stream while the SST - URANS model is likely to be used near the walls. The basic formulation of the incompressible continuity and Navier Stokes equations are

$$
\begin{gathered}
\frac{\partial \overline{u_{i}}}{\partial x_{i}}=0 \\
\frac{\partial \overline{u_{i}}}{\partial t}+\frac{\partial\left(\overline{u_{i} u_{j}}\right)}{\partial x_{j}}=-\frac{1}{\rho} \frac{\partial \bar{p}}{\partial x_{i}}+v \frac{\partial^{2} \overline{u_{i}}}{\partial x_{j}^{2}}+\frac{\partial \tau_{i j}}{\partial x_{j}}=0
\end{gathered}
$$

Where $\tau_{\mathrm{ij}}$ represents the Reynolds stress tensor. The overbar has a different meaning in the LES and SST-URANS formulations. In the URANS regions the overbar represents a time averaged quantity. In this region the Reynolds stress tensor is calculated based on a turbulent viscosity, $\mu_{\mathrm{t}}$, as proposed by Boussinesq,

$$
\begin{aligned}
& \tau_{i j}=-\rho \overline{u_{i} u_{j}}=2 \mu_{t} \bar{S}_{i j}-\frac{2}{3} \delta_{i j} \rho k \\
& \bar{S}_{i j}=\frac{1}{2}\left(\frac{\partial \bar{u}_{i}}{\partial x_{j}}+\frac{\partial \bar{v}_{j}}{\partial x_{i}}\right)
\end{aligned}
$$

which relates the Reynolds stresses to the mean rate of shear tensor. The kinetic energy is calculated using a transport equation.

In the LES regions the overbar in eq.(2) indicates that the velocity component has been filtered in space (the grid size). Here, the Reynolds stress tensor is computed by splitting it into two different parts, the resolved and unresolved part. The resolved part 
can be calculated directly from the resolved part of the fluctuating flow while the effect of the unresolved fluctuations on the main flow is modelled using an eddy-viscosity model.

$$
\tau_{i j}=\rho \overline{u_{i} u_{j}}-\rho \overline{\overline{u_{i}} \bar{u}_{j}}
$$

For higher wavenumbers the filter is larger than the turbulent structures and the so-

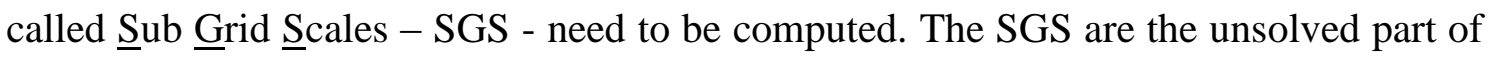
the turbulence in the LES model. In that part of the spectrum the turbulent motion is thought to be isotropic (anisotropy is basically located in the small wave numbers), therefore a simple isotropic model is assumed to be applicable. The unresolved scales are modelled using

$$
\tau_{i j}-\frac{1}{3} \delta_{i j} \tau_{k k}=-2 \mu_{t} \overline{S_{i j}}
$$

where $\bar{S}_{i j}$ is the filtered shear stress tensor, given by

$$
\bar{S}_{i j}=\frac{1}{2}\left(\frac{\partial \overline{u_{i}}}{\partial x_{j}}+\frac{\partial \overline{u_{j}}}{\partial x_{i}}\right)
$$

\section{2 - Detached Eddy Simulation (DES) based on k- $\omega$ SST model}

As mentioned before, the turbulence effects were modelled using a DES model based on a SST - RANS model. This DES model was proposed by Strelets (2001) and the idea behind it is to switch between LES and URANS through blending functions, taking into account the grid size, $\Delta_{\max }$ and the turbulent length scale, $\mathrm{L}_{\mathrm{T}}$. According to Menter (2012),

$$
\begin{aligned}
& C_{D E S} \Delta_{\max }>L_{T} \rightarrow R A N S \\
& C_{D E S} \Delta_{\max }<L_{T} \rightarrow \text { LES }
\end{aligned}
$$

In this formulation $\Delta$ is the maximum edge size of the computational cell domain and $\mathrm{L}_{\mathrm{T}}$ is computed from the kinetic energy and the specific dissipation $\omega$, as follows

$$
L_{T}=\frac{k^{3 / 2}}{\varepsilon}=\frac{k^{3 / 2}}{\beta^{*} k \omega}
$$

This length scale is found in the k-transport equation and is part of the destruction term of $\mathrm{k}$. The specific destruction term, D can be written as,

$$
\begin{aligned}
& D=\varepsilon=\beta^{*} k \omega \\
& D=\frac{k^{3 / 2}}{L_{T}}
\end{aligned}
$$


Using eq (7) to eq. (9), it is possible to rewrite the specific destruction of $\mathrm{k}$ and obtain

$$
D=\frac{k^{3 / 2}}{\min \left(L_{T}, C_{D E S} \Delta\right)}
$$

After some manipulation it is then straightforward to get

$$
D=\frac{k^{3 / 2}}{L_{T}} \max \left(1, \frac{L_{T}}{C_{D E S} \Delta_{\max }}\right)
$$

The term in brackets, called $\mathrm{F}_{\mathrm{DES}}$, is used to pre-multiply the dissipation term in the kequation. When $\mathrm{F}_{\mathrm{DES}}$ is larger than one the rate of destruction of the kinetic energy increases. This, in turn, leads to a decrease in eddy viscosity in the momentum equation inducing the flow to become unsteady.

Menter (2012) slightly changed eq. (11) and introduced the parameter $F_{S S T}$ in order to reduce grid-induced separation, thereby obtaining

$$
D=\frac{k^{3 / 2}}{L_{T}} \max \left(1, \frac{L_{T}}{C_{D E S} \Delta_{\max }}\left(1-F_{S S T}\right)\right)
$$

In the present computations, $\mathrm{F}_{\mathrm{SST}}$ was set to zero so that the original formulation of Strelets (2001) was recovered. The constant $\mathrm{C}_{\mathrm{DES}}$ was set to 0.61 .

\section{4 - AVERAGE QUANTITIES}

\section{1 - Mean axial velocity}

Isolines of the streamwise velocity are shown in Fig. 2. Data were gathered $30 \mathrm{~mm}$ upstream of the outlet and made non-dimensional by using the bulk velocity, $\mathrm{U}_{\text {bulk. }}$ All quantities were averaged over a time of $t=25.40 t_{c}$, where $t_{c}$ is the convective time, obtained by dividing the length of the channel by the bulk velocity.

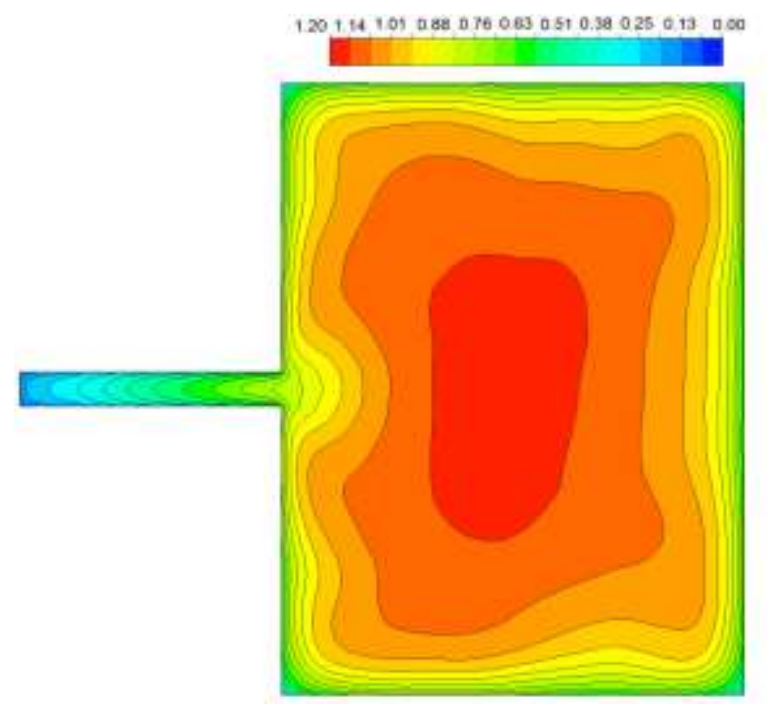

Figure 2 - Isolines of the streamwise velocity component at $\mathrm{x} / \mathrm{L}=0.95$. 
The isolines distribution is quite similar to the one obtained in twin channels connected by a gap. The bulging of the isolines towards the edges is very well pronounced and likely to be caused by the presence of secondary flows at the edges. This effect is strongest at the edges of the wall that is opposite to the gap.

Near the gap, a strong velocity gradient is found and the isoline distribution significantly differs from the opposite wall. While the isolines run almost parallel along the opposite wall, near the slot a significant local bending in the isolines towards the centre of the main channel can be seen. This bending is due to the presence of a strong spanwise velocity component emerging from the slot.

The minor lack of symmetry with respect to the (here horizontal) y-axis is due to the limited averaging time used for the statistical quantities. Inside the main channel the flow is much more unevenly distributed than in the case of twin channels connected by a gap. The maximum velocity on the symmetry line was reached at a distance of nearly $65 \mathrm{~mm}$ from the gap, corresponding to almost one slot depth.

Inside the narrow slot the viscous forces are higher and the velocity is lower than those found in the core of the main channel. The flow decelerates at locations further inside the slot, where it is completely surrounded by three walls. Such a configuration enforces a new mass flow distribution inside the channel, with a slow moving flow in the slot and a faster flow inside the main channel. In Fig. 3 (a) an attempt to fit the velocity distribution near the slot's bottom wall was made. For $\mathrm{y}^{+}>200$ (which is near $\mathrm{y} / \mathrm{p}=$ 0.11) the velocity distribution departs significantly from the law-of-the-wall. In this region a defect layer emerges and the flow is no longer ruled by the presence of the bottom slot wall. Instead, here scales from the defect layer determine the main features of the flow. Moreover, the good agreement between our result and the law-of-the-wall reveals the good quality of our mesh.

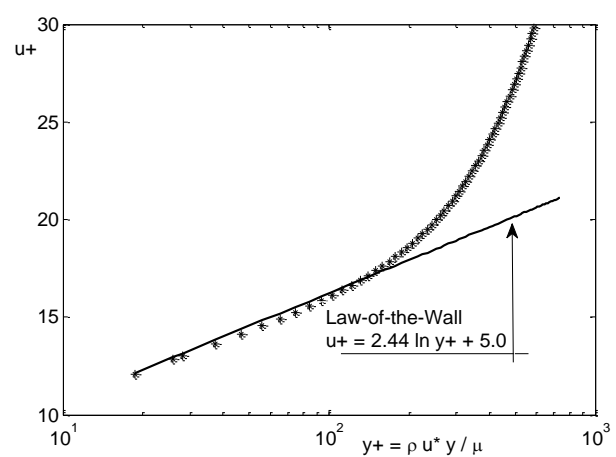

a)

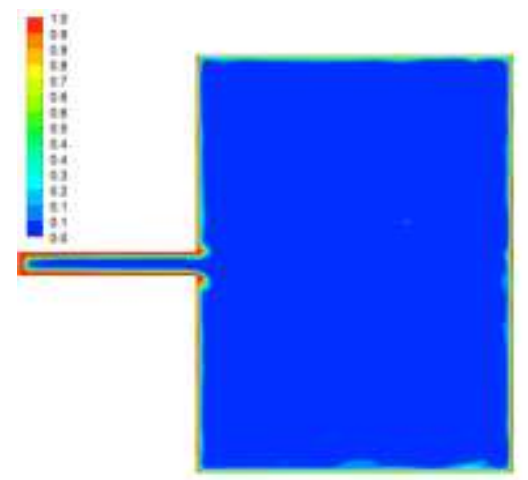

b)

Fig. 3 - a) Law-of-the-wall at the narrow gap. b) contour plot of DES blending function. 
To assess if the designed mesh is able to switch from RANS mode (near wall) and LES mode away from the walls the contour map of DES blending function is shown in Fig. 3 (b). It is possible to see that near the walls the RANS mode prevails (1 indicates second order upwind scheme) whereas, far from the walls the central difference scheme indicates the LES region (0 indicates central difference scheme). At the bottom wall of the slot central difference scheme starts to operate from y+ near to 150 .

\section{2 - Kinetic Energy and Reynold Stress Distribution}

In Fig. 4 (a), (b), (c) and (d), the normal Reynolds stresses (streamwise and spanwise components) and the total turbulent kinetic energy distributions are displayed. The values are made dimensionless using the square of the mean friction velocity, $\mathrm{u}^{* 2}$, computed from the mean shear stress on the top and the bottom walls of the main channels and the density of the fluid (we disregarded the wall shear in the slot). For all quantities the resolved and modelled parts are computed and the dimensionless quantities $\mathrm{k}^{+}, \mathrm{uu}^{+}, \mathrm{vv}^{+}, \mathrm{ww}^{+}$, corresponding to the turbulent kinetic energy, x-normal Reynolds stress, y-normal Reynolds stress and z-normal Reynolds stress, respectively, are obtained.

The highest values of these quantities were found to be located near the gap. The $\mathrm{uu}^{+}$ and $\mathrm{ww}^{+}$peak values were found at the corner of the gap whereas the $\mathrm{vv}^{+}$peak value was located in the middle plane of the channel. The peak values for $\mathrm{uu}^{+} \mathrm{and}_{\mathrm{vv}}{ }^{+}$were both found to be about 11.96, while $\mathrm{ww}^{+}$was found to be only half the $\mathrm{uu}^{+}$and $\mathrm{vv}^{+}$ values, with $\mathrm{ww}^{+}$peak reaching a value of 6.0. The turbulent kinetic energy distribution, $\mathrm{k}^{+}$, very much resembles the distribution of uu+, with peaks in the gap vicinity, the corners and the middle plane of the channel. The peak of $\mathrm{k}^{+}$was found to be 11.50. Compared to Meyers' results for twin channels (Meyer and Rehme, 1994), $\mathrm{k}^{+}$and $\mathrm{ww}^{+}$ were found to be increased by $25 \%$, while the difference in $u^{+}$was only $3 \%$.

Unfortunately, the authors did not provide these quantities for a more detailed quantitative comparison. 


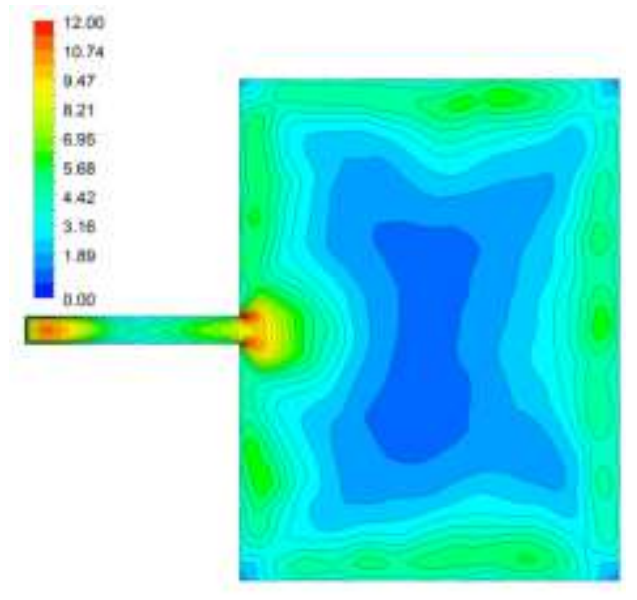

a)

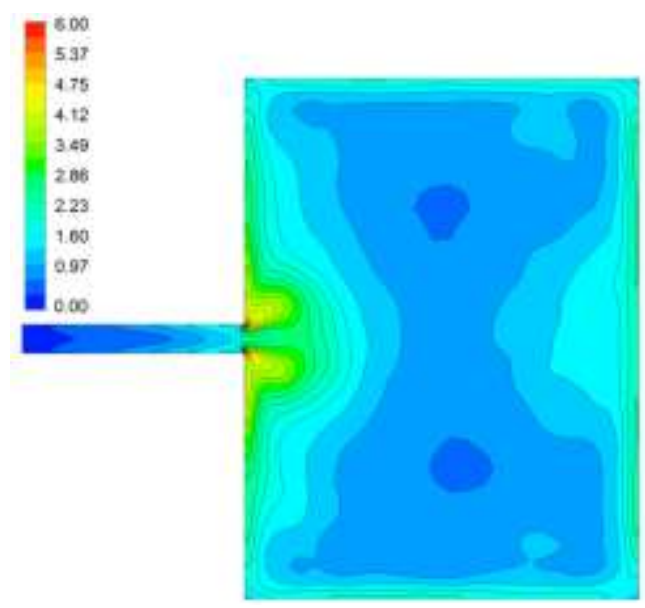

c)

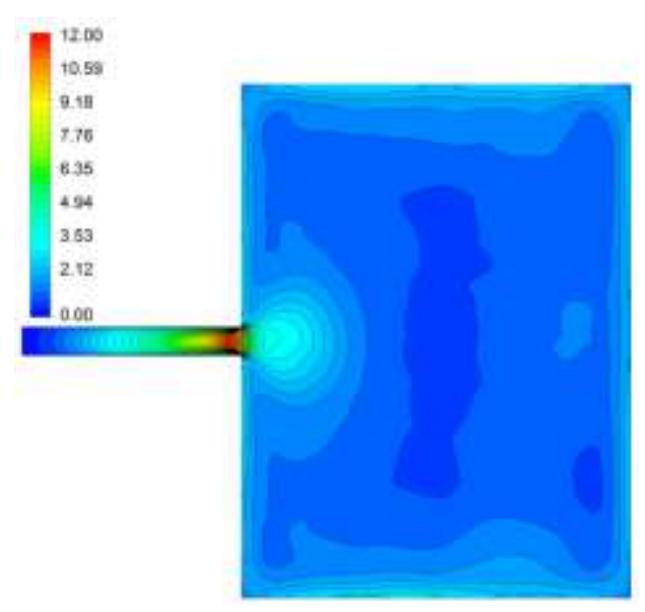

b)

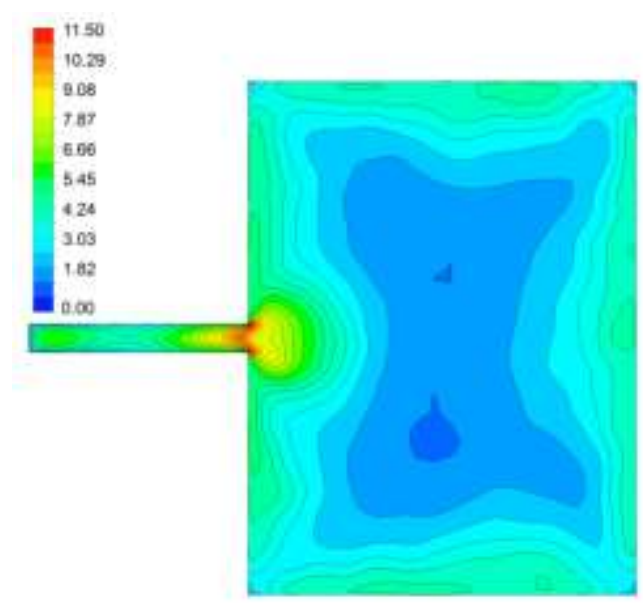

d)

Fig. 4 - Maps of turbulent quantities distribution. (a) streamwise normal Reynolds tress $-\mathrm{uu}^{+}$.(b) spanwise Reynolds stress $-\mathrm{vv}^{+}$. (c) Reynolds tress $-\mathrm{ww}^{+}$. d) turbulent kinetic energy, $k_{T}^{+}=k_{R E S}^{+}+k_{M O D}^{+}$.

Qualitatively, however, they seem to be in fair agreement with Meyer's results, with the exception of the results obtained deeper inside the slot. There, the contours were found to differ due to the slot's bottom wall. Inside the main channel the contours are quite similar to the ones found in square channels. The isolines of the kinetic energy are seen to bulge towards the corners indicating secondary flows at these locations.

From Fig. 4 (a), (b) and (c) it is important to note the anisotropy of normal Reynolds stresses, especially inside the slot. Due to the small width of the slot, the mixing rate normal to the upper and lower walls of the slot tends to be reduced in comparison with streamwise and spanwise mixing rates. It is worth mentioning that the streamwise and spanwise normal Reynolds stress components account for more than 90 $\%$ of the total kinetic energy inside the small subchannel, decreasing to $65 \%$ at the 
middle of the main channel. This fact implies that the flow and the turbulence inside the slot are predominantly two-dimensional. In fact, the distribution of the total turbulent averaged quantities - i.e $\mathrm{k}^{+}, \mathrm{uu}^{+}, \mathrm{vv}^{+}, \mathrm{wv}^{+}$- along the symmetry line in Fig. 5 (a) shows that the z-normal Reynolds stress $\mathrm{ww}^{+}$is much smaller than $\mathrm{uu}^{+}$and $\mathrm{vv}^{+}$.

In Fig.5 (b) the average quantity of $\mathrm{uv}^{+}\left(\overline{u^{\prime} v^{\prime} / \mathrm{u}^{* 2}}\right)$, is plotted along the symmetry line against the non-dimensional y-coordinate.

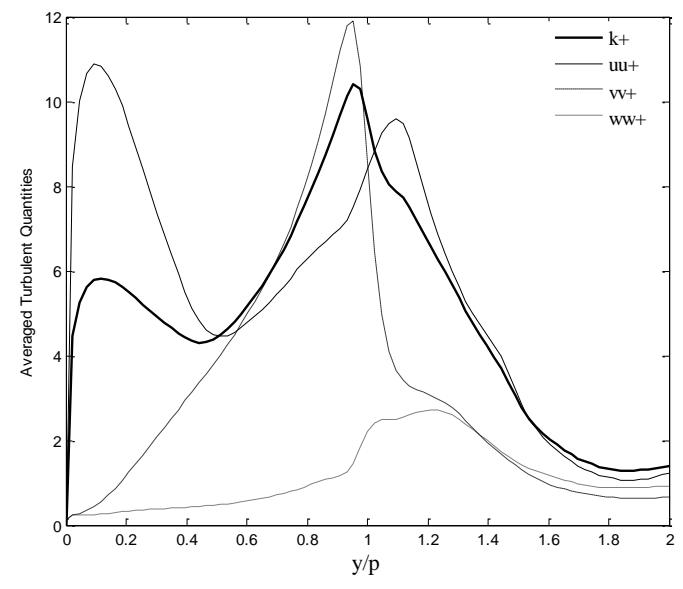

a)

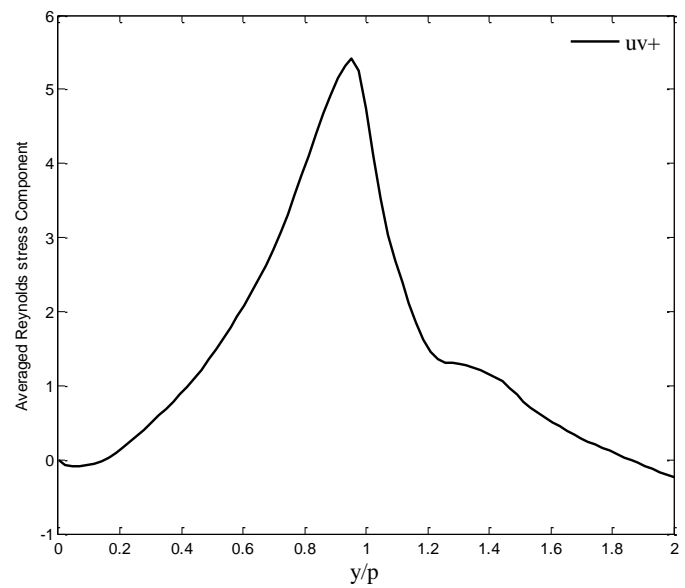

b)

Fig. 5 - (a) Averaged turbulent quantities along the symmetry line. (b) Reynolds stress.

This quantity departs from zero at $y / p=0.11$, reaches its maximum value where the slot and main channel meet and then decreases again to zero at $y / p=1.80$, where the axial velocity is maximum. The other two quantities, $\mathrm{uw}^{+}=\overline{u^{\prime} w^{\prime}} / \mathrm{u}^{* 2}$ and $\mathrm{vw}^{+}=\overline{v^{\prime} w^{\prime}}$ $/ \mathrm{u}^{* 2}$, show very small peaks on the symmetry line, as high as 0.18 and 0.11 , respectively. On the other hand, at the corners of the gap, two peaks of both quantities were found, one being positive and the other one negative. For both quantities the peaks are about \pm 3.05 . Positive and negative peaks of these quantities at the corners of the gap of the twin channels were previously reported by Meyer and Rehme (1994).

\section{5 - STRUCTURE OF TURBULENT FLOW IN THE CHANNEL}

\section{1 - Quasi-periodic Patterns}

One of the most remarkable features of the turbulent flow in open or closed compound channels is the quasi-periodic patterns of velocity. Such behaviour has been very well known for at least 40 years and has led to a significant research activity since then. Over the years, the main target was to build a picture of the turbulent flow inside the channel by using time-traces of the velocities and assessing the main frequency, the convection velocity, wavelengths and the mixing rates yielded by the flow patterns. 
Meyer and Rehme (1994) were able to explain the flow patterns observed by the presence of two counter-rotating vortices on each side of the gap that are able to cross the narrow gap, thereby increasing the local mixing rate.

Figures 6 and 7 show time traces of the stream and spanwise velocity components along the symmetry line, ranging from $y / p=0.125$ up to $y / p=1.87$.

It can be seen that both time-trace components show the existence of quasi-periodic patterns in the region ranging from deep inside the slot towards the main channel. However, the time-traces can be seen to quickly lose their sinusoidal structure as soon as the probes leave the slot and enter the main channel. This characteristic is in fair agreement with the experimental work of Goulart et al. (2013). The plots are labeled by the relative depth $y / p$ of the probe in the slot. Both figures 6 and 10 follow the same sequence of probe locations.
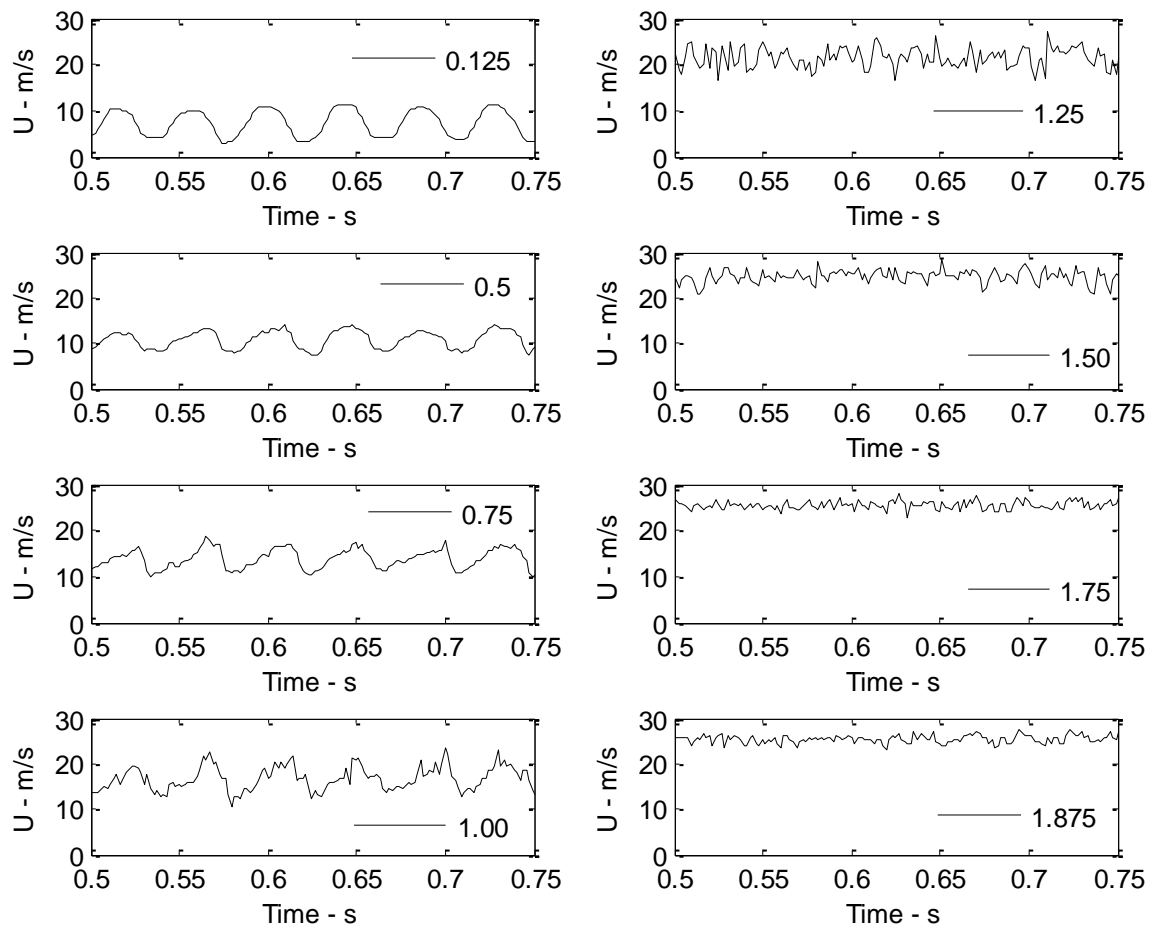

Fig 6 - Time-traces of streamwise velocity component at $\mathrm{y} / \mathrm{p}=0.125,0.5,0.75,1.0$, $1.25,1.50,1.75$ and 1.87 . 

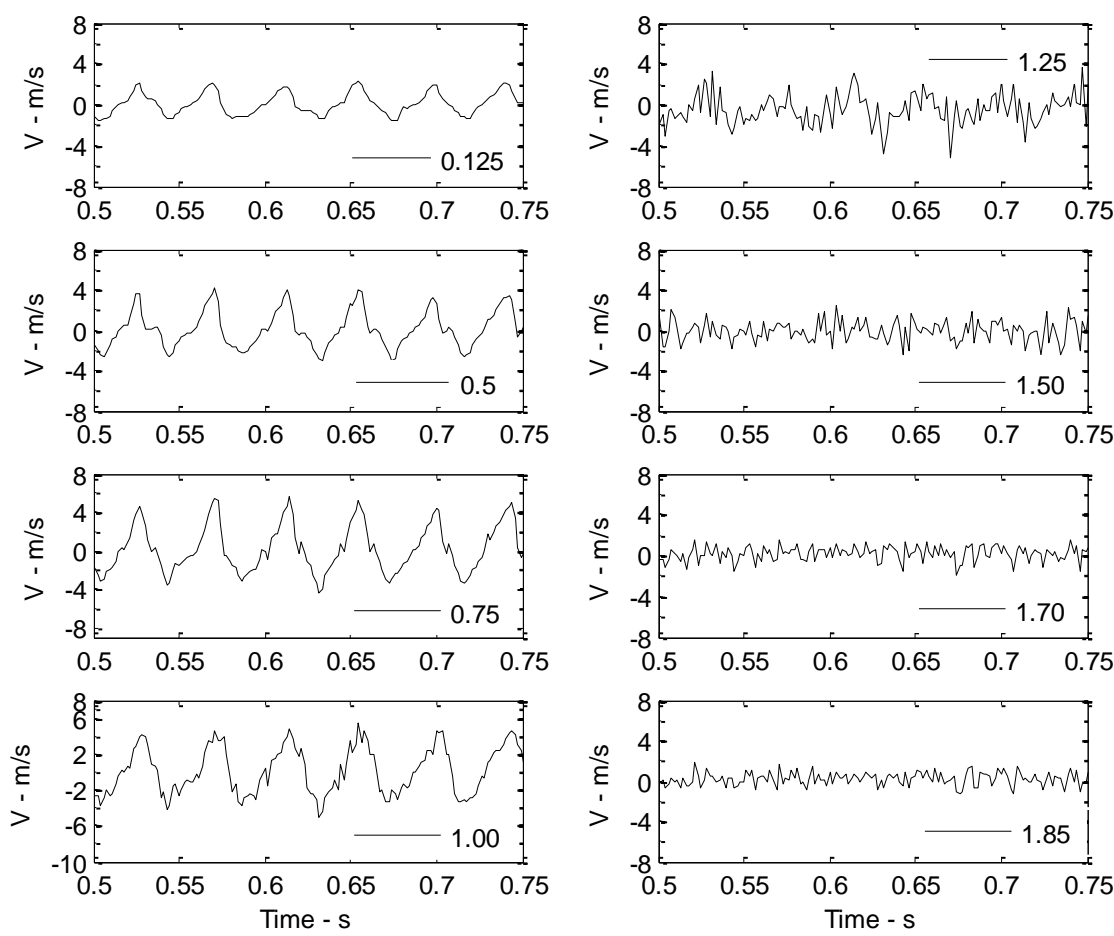

Fig 7 - Time-traces of spanwise velocity component at $y / p=0.125,0.5,0.75,1.0$,

$$
1.25,1.50,1.75 \text { and } 1.87 \text {. }
$$

The main frequency of these velocity time-traces was estimated by performing a temporal auto-correlation,

$$
\operatorname{Coeff}(x, t)=\frac{u(t+\Delta t) * u(t)}{u^{2}(t)}
$$

Figures 8 and 9 show the time correlations for the velocity series at various stations $y / p$ along the symmetry line. The number assigned to each graph on the top right corner indicates the relative position along the symmetry line.

The correlation coefficients reach their maximum values inside the slot and subsequently decrease towards the main channel implying that the large scale structures in the flow mainly dominate the small subchannel flow. When the probe moves towards the main channel incoherent motions (quasi-random fluctuations) become superimposed on the velocity signals attributing to an increasing non-smoothness of the autocorrelation signals. Despite these little changes the time interval between two statistically correlated peaks remains constant at $0.042 \mathrm{~s}$, implying that the main frequency associated with the large scales structures is $23.80 \mathrm{~Hz}$. This frequency matches the one found by Meyer and Rehme (1994) using hot wire probes. 

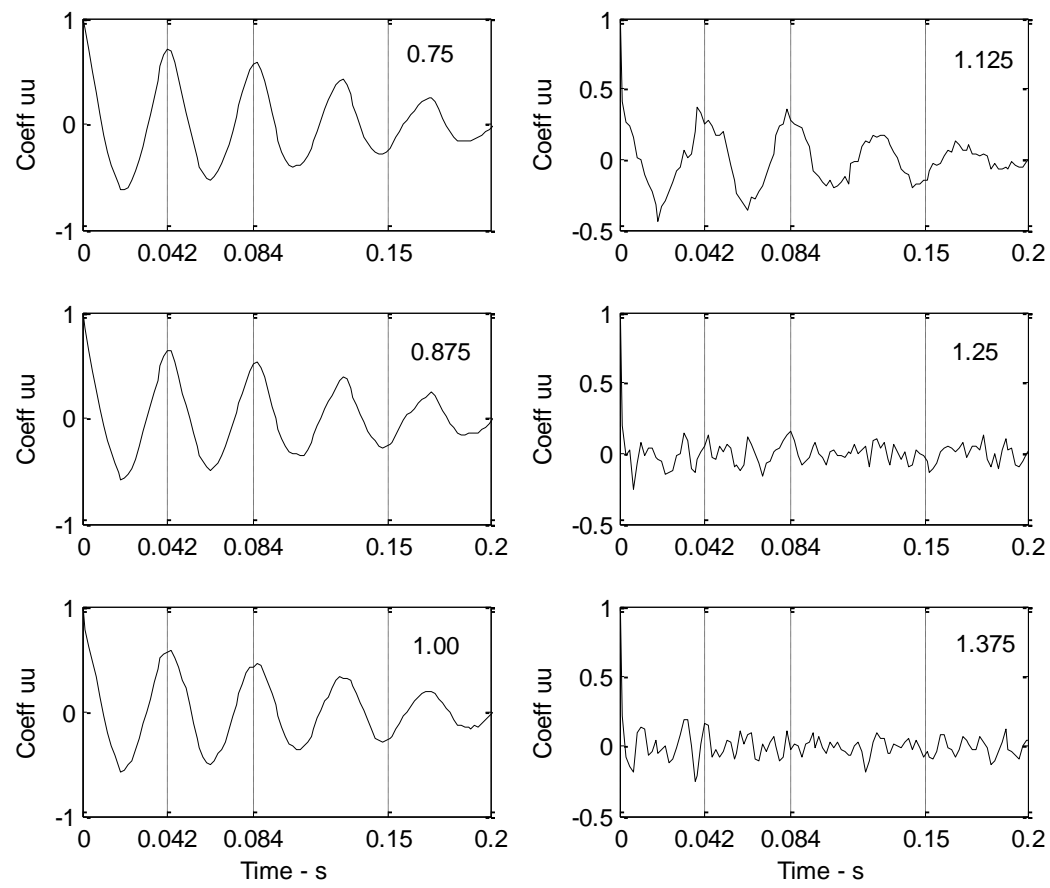

Figure 8 - Time correlation coefficients of the streamwise velocity along the symmetry line.

The authors measured the main frequencies in this kind of channel and plotted the results against Reynolds number. The experimental main frequency was found to be $24.61 \mathrm{~Hz}$ for a Reynolds number of $2.24 \times 10^{5}$. Only a slight difference can be seen in the time correlation of both velocity components. In general, the spanwise component of the velocity fluctuation is better correlated inside the small subchannel than the streamwise component. However, as shown in Fig. 9, the periodic pattern vanishes soon after $\mathrm{y} / \mathrm{p}=1.125$. 

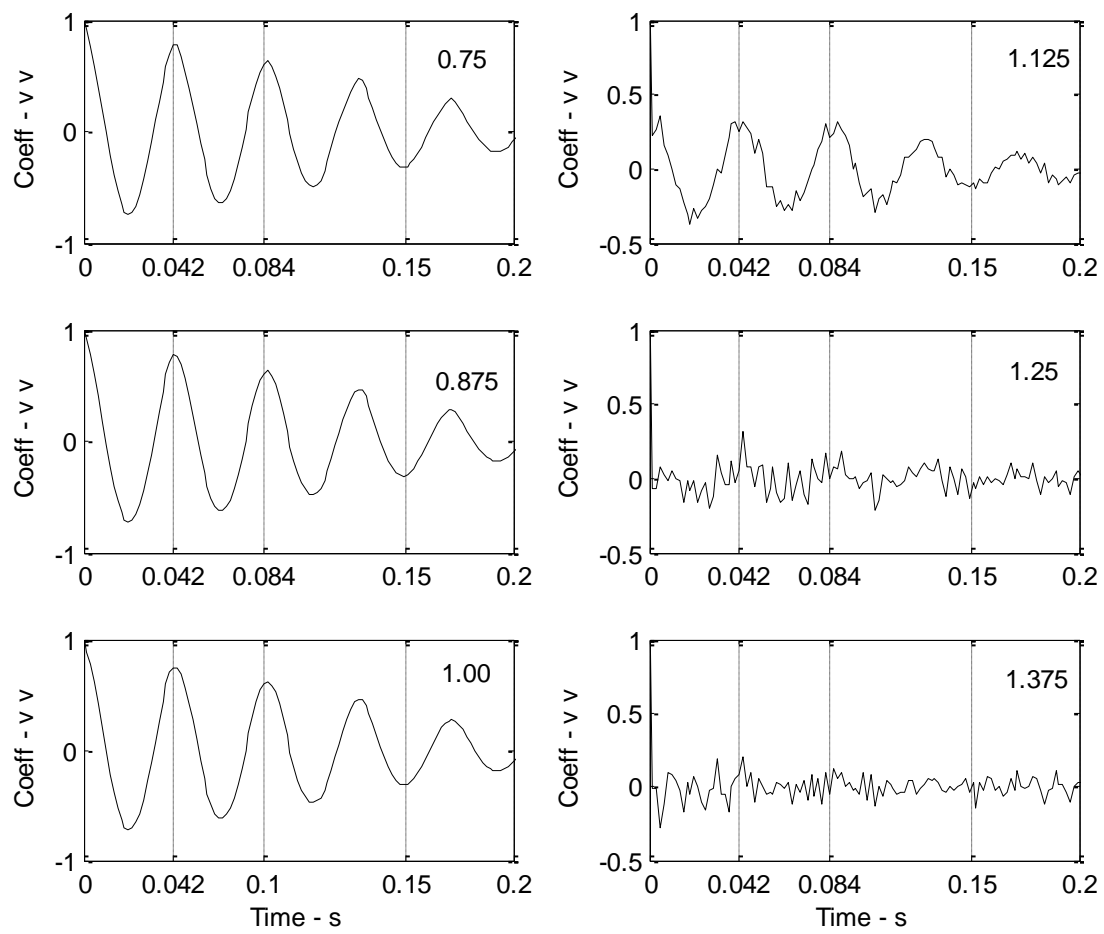

Figure 9 - Time correlation coefficients for the streamwise velocity along the symmetry line at $\mathrm{y} / \mathrm{p}=0.75,0.875,1.0,1.125,1.25,1.375$.

Cross correlations were also performed using both velocity components with one probe fixed at the edge $(y / p=1)$ and the other free to move in and out of the small subchannel. The cross correlation functions were assigned using the distance between both probes and the depth of the small channel, $\Delta y / p$. These non-dimensional coordinates are $(+)$ inside the main channel and (-) in the small subchannel. Note that the fixed probe is located at $\Delta \mathrm{y} / \mathrm{p}=0$.

Figure 10 (a) and (b) show the cross correlation in time for the $u$ and v-velocities.

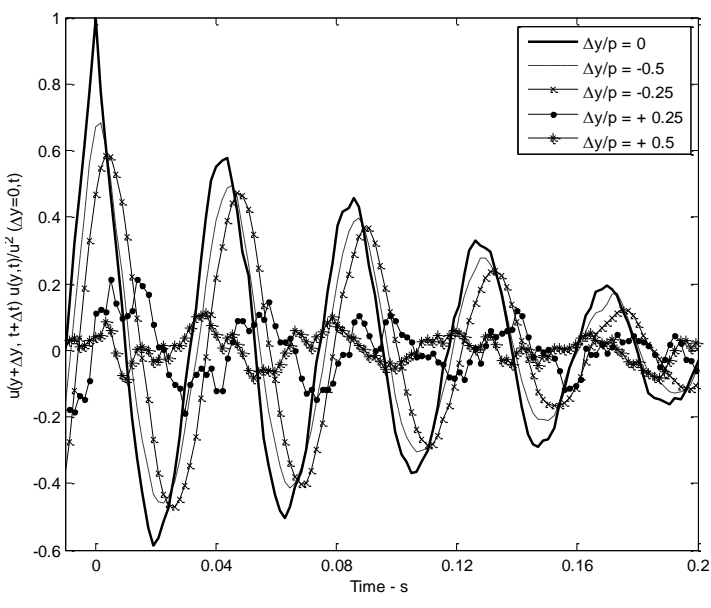

a)

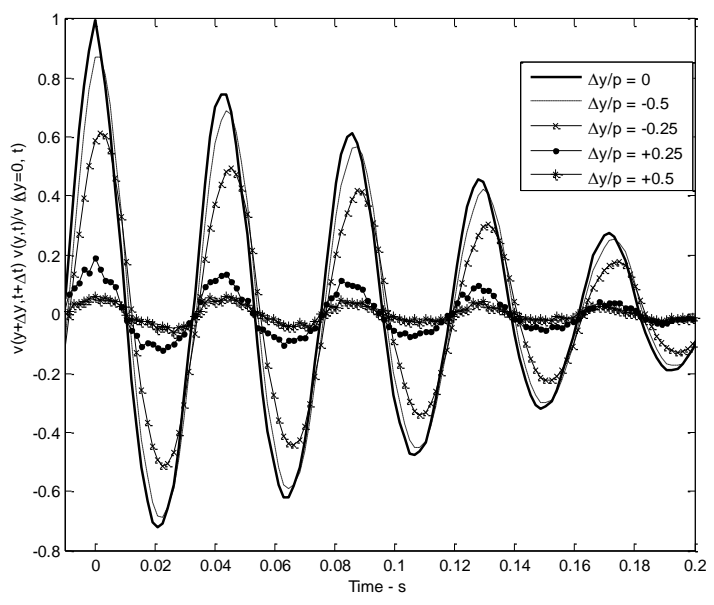

b)

Figure 10 - Cross time correlations. a) streamwise velocity component. b) spanwise velocity component. 
Both components are advanced in time inside the gap. For the spanwise component the time shift is found to be virtually negligible. On the other hand, for the u-velocity component the time shift increases with distance between points, resulting in a maximum phase shift of $\pi / 3$. This result differs from the one obtained for twin-channels connected by a gap or tight-lattice fuel bundle. There, a phase shift of $180^{\circ}$ was found supporting the model proposed by Möller (1991) that there are two arrays of counterrotating vortices on each side of the gap.

From this, we can conclude that there is only one array of large vortices present in the slot and the model proposed by Möller (1991) does not fit this single channel-slot interface case. The bottom wall of the small subchannel avoids the formation of a second mixing layer that would result in a second array of counter rotating vortices emerging. This result seems to support our main idea that these large scale structures arise from the main flow instability that is characterized by an inflexional velocity profile.

To give a more complete picture of turbulent flow structures we also performed spatial correlations along the main axis of the channel. The idea behind this calculation is to assess the axial wavelength, $\lambda$, and compare it to the result provided by Meyer and Rehme (1995). Figure 11 shows cross-correlation functions for both components. The time-traces were gathered at the gap region $(\mathrm{y} / \mathrm{p}=1)$. The wavelength can again be assessed by determining how far the velocity fluctuations signals are correlated.

The wavelength associated to the vortex formation was calculated to be $360 \mathrm{~mm}$, corresponding to $\lambda / p=4.67$. This result is in fair agreement with Meyer and Rehme's measurement, Fig. 11 (b). Despite some scattering of the data the authors found a nondimensional wavelength $\lambda / \mathrm{p}=4.50$ for this kind of channel (the authors used $\mathrm{d} / \mathrm{g}$ as dimensionless parameter of the small slot, $d$ being the deepness and $g$ the width).

Figure 11 (c) shows instantaneous spanwise velocity contours in the middle plane of the channel. An array of large counter-rotating vortices can be seen (moving) in the streamwise direction. These structures are carried by the main flow inside the slot with a certain convection velocity, $U_{c}$. The convection velocity can be determined using only the wavelength and the main frequency of the large vortices,

$$
U_{c}=\lambda f
$$

yielding a convection velocity, $U_{c}$ normalized by edge velocity, of $U_{c} / U_{\text {edge }}=0.52$. This number slightly underpredicted in comparison with that found experimentally by Meyer 
and co-worker (Meyer and Rehme, 1994), whose non-dimensional result was $\mathrm{U}_{\mathrm{c}} / \mathrm{U}_{\text {edge }}=$ 0.57 .

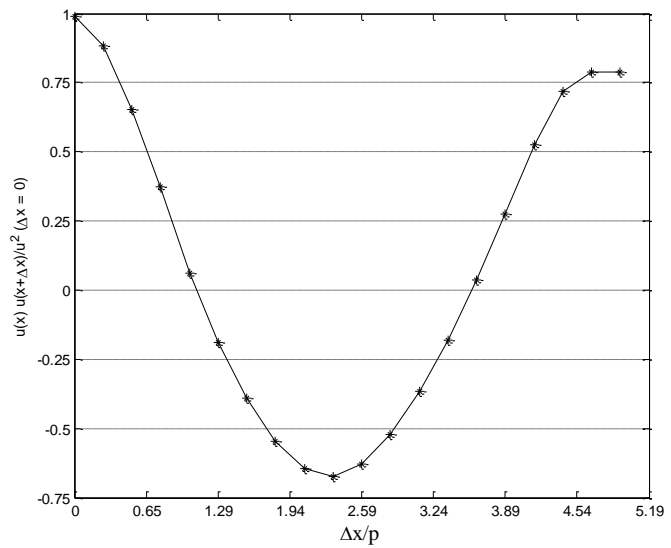

a)

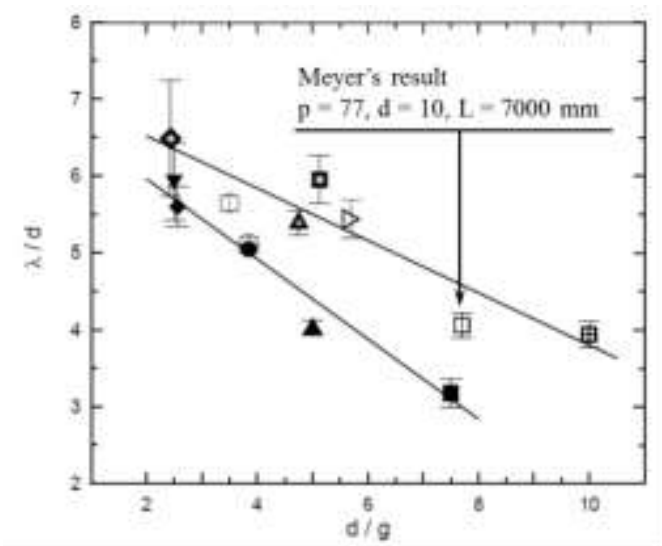

b)

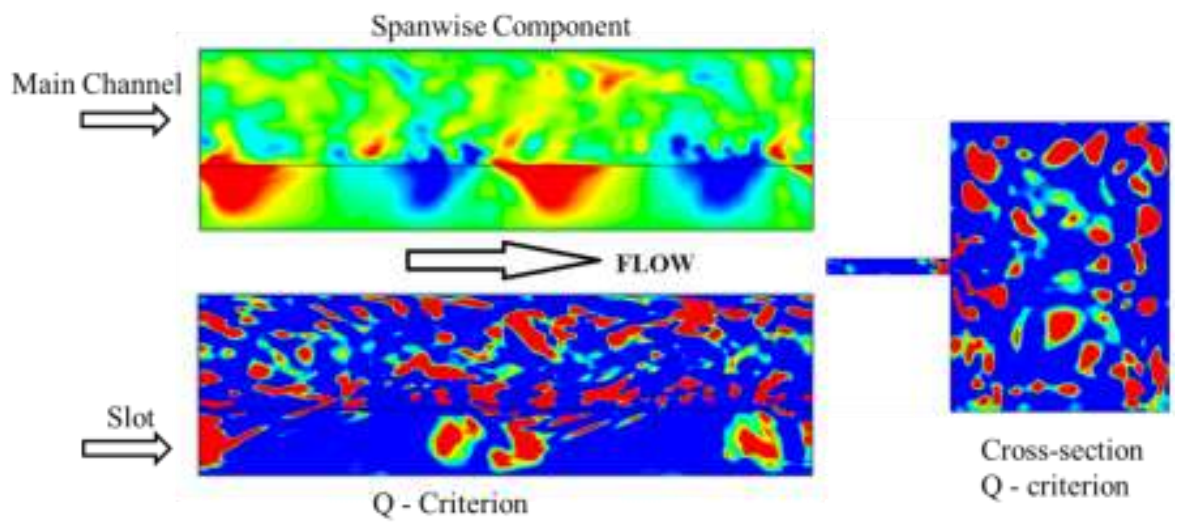

c)

Figure 11 - Spatial correlation for streamwise velocity along the main channel's axis. b) non-dimensional wavelength plotted against p/d-ratio (Meyer and Rehme, 1995). c) large vortices traveling downstream and Q-criterion (at $\mathrm{t}=21$ flow-through times).

The iso-contours of the Q-criterion, proposed by Hunt et al.(1988), are also displayed in Fig. 11 (c), being given by

$$
Q=\frac{1}{2}\left(\omega^{2}-S^{2}\right)
$$

The value is positive when the vorticity magnitude surpasses the magnitude of the shear strain rate, identifying the locations where vorticity dominates shear. In Fig. 11 (c) the instantaneous contours of Q are shown and a threshold for Q is applied. Iso-contours are shown only for $\mathrm{Q} \geq 1 \mathrm{~s}^{-2}$. 
In the middle plane of the channel (along the $x$-axis) we can see structures showing a high coherence inside the gap. These structures seem to appear in pairs, leaving a significant gap between each new pair. This kind of motion leaves us to think that some intermittent processes may happen, which are outside the scope of this work. Such intermittent processes have already been reported in a close compound channel by e.g. Chouring and Tavoularis, 2014.

The Q-criterion applied in the cross-section shows very well formed structures spreading out in the main channel. At the edges, mainly at the top corners and the lower right corner, it is possible to see the organized structures of the secondary turbulent flow. They also appear near the gap, explaining the bulging of the kinetic isolines in this region of the channel.

\section{6 - CONCLUDING REMARKS}

In this paper, we simulated turbulent isothermal flow in a periodic channel connected to a rectangular slot by using ANSYS ${ }^{\circledR}$ CFX. Hybrid model called DES-SST was used to account for turbulence effects. This model switches between URANS and LES, whichever is more appropriate. The main goal of our simulation was to provide quantitative data on the turbulence characteristics in a single channel with a lateral slot. The results are compared to results provided by Meyer and Rehme (1994) (MR). Despite MR focusing mainly on twin channels, they also provided some results from the dynamic of the flow for single channels.

Turbulent statistical quantities such as the turbulent kinetic energy, $\mathrm{k}^{+}$and the normal Reynolds stresses were found to reach their maximum values in the vicinity of the slot. These quantities were $25 \%$ higher in comparison with the twin channels results of Meyer and Rehme, with the exception of the normal Reynolds stress, uu ${ }^{+}$, which was found to be only $3 \%$ larger than the experimental data in twin channels. The small width of the slot was found to force the flow inside it to be virtually two-dimensional as can be seen by the significant contribution of the streamwise and spanwise components of the normal Reynolds stresses to the total amount of kinetic energy.

In regard to the structure of the turbulent flow, large eddies were seen to dominate in the small slot. Because of these vortices, the time-traces of the velocity in the slot were found to exhibit clear sinusoidal patterns These patterns were also found to extend slightly into the main channel, up to about $y / p=1.125$. Qualitatively, the simulation results were found to be in fair agreement with the few results published for this kind of 
channel by Meyer and Rehme (1994). The frequency and the wavelength of the large scale structures were successfully reproduced. The convection velocity of the large vortices was slightly overpredicted by a factor of less than $10 \%$ in comparison with experimental values. The spatial velocity correlations and the Q-criterion visualization made it possible to verify the applicability of the model proposed by Möller (1991) for the flow in twin channels connected by a gap. It was found that this model is not valid for the flow in a single channel with a gap.

The strategy of performing the calculations using periodic boundary conditions in the streamwise direction made it possible to significantly shorten the channel length of the original problem: from $7000 \mathrm{~mm}$ to $730 \mathrm{~mm}$. We found that, counter to the conclusion of Chang and Tavoularis (2012), periodic boundaries can be successfully used to simulate compound channel flows provided that the length of the computational domain is sufficient to accommodate the important streamwise structures. The quantitative results appeared not to be affected by the proposed technique.

\section{ACKNOWLEDGEMENTS}

The first author would like to thank the Brazilian National Research Council - CNPq, for supporting him during this research. The authors also acknowledge the support provided by Brunel University London during the development of this research project.

\section{7 - NOMENCLATURE}

$\mathrm{p} \quad$ depth of small subchannel, $\mathrm{m}$

d width of small subchannel, $m$

W width of main channel, $\mathrm{m}$

$\mathrm{H} \quad$ height of main channel, $\mathrm{m}$

$\mathrm{D}_{\mathrm{h}} \quad$ hydraulic-diameter, $\mathrm{m}$

L length of computational domain, $\mathrm{m}$

$\operatorname{Re} \quad$ Reynolds number, $\operatorname{Re}=\frac{U_{b u l k} D_{h}}{v}$

Coeff coefficient of correlations

f frequency, $\mathrm{Hz}$

$\mathrm{t} \quad$ time, $\mathrm{s}$ 


$$
\begin{aligned}
& \text { Q Q-criterion, 1/s } \\
& \mathrm{U}_{\mathrm{c}} \quad \text { convection velocity of the large vortices, } \mathrm{m} / \mathrm{s} \\
& U_{\text {bulk }} \quad \text { bulk velocity, } \mathrm{m} / \mathrm{s} \\
& \dot{m} \quad \text { mass flow rate, } \mathrm{kg} / \mathrm{s} \\
& \mathrm{t}_{\mathrm{c}} \quad \text { convective time, } \mathrm{s}-\mathrm{L} / U_{\text {bulk }} \\
& \mathrm{uu}^{+}, \mathrm{vv}^{+}, \mathrm{ww}^{+} \text {dimensionless normal Reynolds stresses, } \overline{u^{\prime} u^{\prime}} / \mathrm{u}^{* 2}, \overline{v^{\prime} v^{\prime}} / \mathrm{u}^{* 2}, \overline{w^{\prime} w^{\prime}} / \mathrm{u}^{* 2} \\
& \mathrm{uv}^{+}, \mathrm{uw}^{+}, \mathrm{vw}^{+} \text {dimensionless Reynolds stresses, } \overline{u^{\prime} v^{\prime}} / \mathrm{u}^{* 2}, \overline{u^{\prime} w^{\prime}} / \mathrm{u}^{* 2}, \overline{v^{\prime} w^{\prime}} / \mathrm{u}^{* 2} \\
& \mathrm{k}^{+} \quad \text { dimensionless total turbulent kinetic energy, } k_{T} / \mathrm{u}^{* 2} \\
& \mathrm{k}_{\mathrm{Coh}}, \mathrm{k}_{\mathrm{nc}} \quad \text { Coherent and non-coherent part of turbulent kinetic energy, } \mathrm{m}^{2} / \mathrm{s}^{2} \\
& \mathrm{u}^{* 2} \quad \text { square of velocity friction, } \mathrm{m}^{2} / \mathrm{s}^{2}-\tau_{\text {wall }} / \rho \\
& \mathrm{x}, \mathrm{y}, \mathrm{z} \quad \text { coordinates, } \mathrm{m} \\
& \Delta \mathrm{x} \quad \text { streamwise grid size, } \mathrm{m} \\
& \mathrm{y}^{+} \quad \text { Wall coordinate, } y u^{*} / \mathrm{v}
\end{aligned}
$$

Greek symbols

$$
\begin{array}{ll}
\rho & \text { density of the fluids, } \mathrm{kg} / \mathrm{m}^{3} \\
\mu & \text { dynamic viscosity, Pa.s } \\
\nu & \text { kinematic viscosity, } \mu / \rho, \mathrm{m}^{2} / \mathrm{s} \\
\lambda & \text { wavelength distance between two consecutive vortices with same sign, } \mathrm{m}
\end{array}
$$

\section{8 - REFERENCES}

Chang, D and Tavoularis, S, 2005, "Unsteady numerical simulations of turbulence and coherent structures in axial flow near a narrow gap,” J. Fluids Eng. 127, pp. 458-466.

Chang, D and Tavoularis, S, 2008, "Simulations of Turbulence, heat transfer and mixing across narrow gaps between rod-bundle subchannels," Nuclear Engineering and Design, 238, pp. 109-123.

Chang, D and Tavoularis, S, 2012, “ Numerical simulation of developing flow and vortex street in a rectangular channel with a cylindrical core, "Nuclear Engineering and Design, 243, pp. 176-179. 
Choueiri, G. H. and Tavoularis, S., 2014, "Experimental investigation of flow development and gap vortex street in an eccentric annular channel - Part 1," J. Fluids Mech. 752, pp. 521-542.

Derksen, J. J, 2010 "Simulations of lateral mixing in cross-channel flow," Computers \& Fluids 39, pp. 1058-1069.

Goulart, J. N. V. and Möller, S. V., 2007, "Shear Flow in Compound Channels," In: 19th International Congress of Mechanical Engineering, Brasília, Brazil.

Goulart, J, N. V., Noleto, L, G. and Möller, S, V, 2013, "Experimental Study of Mixing Layer in a Closed Compound Channel," Journal of the Brazilian Society of Mechanical Sciences and Engineering (print), 36, p. 411-420.

Guellouz, M.S. and Tavoularis, S., 2000a, "The structure of the turbulent flow in a rectangular channel containing a single rod - Part 1: Reynolds-Average measurements," Experimental Thermal and Fluid Science, 23, pp.59-73.

Home, D., Arvanitis, G., Ligtstone, M.F. and Hamed, M.S., 2009, "Simulation of flow pulsation in a twin rectangular sub-channel geometry using unsteady Reynolds averaged Navier-Stokes modelling,” Nucl. Eng. Des. 239, 2964-2980.

Home, D., Lightstone, M. F., 2014, "Numerical investigation of quasi-periodic flow and vortex structure in a twin rectangular subchannel geometry using detached eddy simulation," Nuclear Engineering and Design, 270, 1-20.

Hooper, J.D. and Rehme, K., 1984, "Large-scale structural effects in developed turbulent flow through closely-spaced rod arrays,” J. Fluid Mech. 145, 305-337.

Hunt, J., Wray. W. W. and Moin, P., 1988, "Eddies, streams and convergence zones in turbulent flows," Report CTR-S88. Center of Turbulence Research, Stanford University.

Rehme, K. and Meyer, L., 1998, "Experimental investigation of turbulent transport of momentum and energy in a heated rod bundle," Nuclear Engineering and Design, 180, 185-206.

Meyer, L. and Rehme, K., 1994, "Large-scale turbulence phenomena in compound rectangular channels," Experimental Thermal and Fluid Science, 8,pp. 286-304.

Meyer, L. and Rehme, K., 1995, "Periodic vortices in flow though channels with longitudinal slots or fins," 10th Symposium on Turbulent Shear Flows, The Pennsylvania State University, University Park, August 14-16.

Menter, F., Ferreira, C., J., Esch, T. and Konno, B., 2003, "The SST Turbulence Model with Improved Wall Treatment for Heat Transfer Predictions in Gas Turbines," In: Proceedings of the International Gas Turbine Congress, Tokyo, Japan.

Menter, F., R., 2012, "Best Practice: Scale-Resolving Simulations in ANSYS ${ }^{\circledR}$ CFD," ANSYS $^{\circledR}$, Germany. 
Merzari, E., Ninokata, H. and Baglietto, E., 2008a, "Numerical simulation of flows in tight-lattice fuel bundles,” Nucl. Eng. Des. 238, 1703-1719.

Meyer, L., 2010, "From Discovery to Recognition of Periodic Large Scale Vortices in Rod Bundles as Source of Natural Mixing Between Subchannels-A review," Nuclear Engineering and Design, 240, pp. 1575 - 1588.

Möller, S. V., 1991, “On Phenomena of Turbulent Flow through Rod Bundles,”. Experimental Thermal and Fluid Science, 4, pp. 25-35.

Rehme, K., 1992, "The structure of turbulence in rod bundles and the implication on natural mixing between subchannels,” Int. J. Heat Mass Transfer, 35, pp: 567-581

Rowe, D.S., Johnson, B.M. and Knudsen, J. G., 1974, "Implications concerning rod bundle crossflow mixing based on measurements of turbulent flow structure," Int. J. Heat Mass Transfer, 17, pp. 407-419.

Strelets, M., 2001, "Detached Eddy Simulation of massively separated flows". AIAA Paper 2001-0879, Reno, NV.

Tavoularis, S., 2011, "Rod bundle vortex networks, gap vortex streets, and gap instability: A nomenclature and some comments on available methodologies," Nucl. Eng. Des. 241, 2624-2626.

Wu, X. and Trupp, A. C., 1994, "Spectral measurements and mixing correlations in a simulated rod bundle subchannels," Int. J. Heat Transfer, 37, pp. 1277-1281. 